\title{
Recent Progress on Submersions: A Survey and New Properties
}

\author{
Gabriel Picavet \\ Université Blaise Pascal, Laboratoire de Mathématiques UMR 6620 CNRS, Les Cézeaux, 24 Avenue des Landais BP 80026, \\ 63177 Aubière Cedex, France \\ Correspondence should be addressed to Gabriel Picavet; picavet.gm@wanadoo.fr
}

Received 27 November 2012; Accepted 15 March 2013

Academic Editor: Prasanna Kumar Sahoo

Copyright (C) 2013 Gabriel Picavet. This is an open access article distributed under the Creative Commons Attribution License, which permits unrestricted use, distribution, and reproduction in any medium, provided the original work is properly cited.

\begin{abstract}
This paper is a survey about recent progress on submersive morphisms of schemes combined with new results that we prove. They concern the class of quasicompact universally subtrusive morphisms that we introduced about 30 years ago. They are revisited in a recent paper by Rydh, with substantial complements and key results. We use them to show Artin-Tate-like results about the 14th problem of Hilbert, for a base scheme either Noetherian or the spectrum of a valuation domain. We look at faithfully flat morphisms and get "almost" Artin-Tate-like results by considering the Goldman (finite type) points of a scheme. Bjorn Poonen recently proved that universally closed morphisms are quasicompact. By introducing incomparable morphisms of schemes, we are able to characterize universally closed surjective morphisms that are either integral or finite. Next we consider pure morphisms of schemes introduced by Mesablishvili. In the quasicompact case, they are universally schematically dominant morphisms. This leads us to a characterization of universally subtrusive morphisms by purity. Some results on the schematically dominant property are given. The paper ends with properties of monomorphisms and topological immersions, a dual notion of submersions.
\end{abstract}

\section{Introduction}

Our aim is to give a survey on recent progress on submersions and new results that commutative algebraists may find useful. We also recall results that are needed. The paper is written in the language of schemes because it is sometimes necessary to enlarge the category of commutative rings to get proofs, but the results can be easily translated.

Submersive morphisms of schemes (or submersions) $f$ : $X \rightarrow Y$ are surjective morphisms inducing the quotient topology on $Y$; that is, $Z \subseteq Y$ is an open (closed) subset if and only if $f^{-1}(Z)$ is open (closed). They are also called topological epimorphisms by some authors like Voevodsky who defines and uses the $h$ and $q f h$-(Grothendieck) topologies [1]. They appear naturally in many situations such as when studying quotients, homology, descent, and the fundamental group of schemes. A morphism of schemes $f: X \rightarrow Y$ is called universally submersive if $X \times_{Y} Y^{\prime} \rightarrow Y^{\prime}$ is submersive for each morphism $Y^{\prime} \rightarrow Y$. The first proper treatment of submersive morphisms was settled by Grothendieck, with applications to the fundamental group of a scheme. We singled out a subclass of submersive morphisms in [2] and dubbed them subtrusive morphisms (or subtrusions). Submersive morphisms used in practice are subtrusive. Our study was established in the affine schemes context. But as Rydh showed, the theory can be extended to the arbitrary schemes context [3]. Over a locally Noetherian scheme, a universally submersive morphism is universally subtrusive, showing again that the class of subtrusive morphisms is natural. In this section, we give information about our aims, notational conventions, and definitions, recalling results that are needed in the other sections.

We recall the following facts.

The closed subsets of the constructible topology on a scheme (cf. [4, I.7.2]) (also termed the patch topology) are the proconstructible subsets (see [4, I, Section 7]).

The Zariski topology induces a partial ordering on the underlying set of points of a scheme $X$. We let $x \leq x^{\prime}$ if $x \in \overline{\left\{x^{\prime}\right\}}$, that is, if $x$ is a specialization of $x^{\prime}$, or equivalently, if $\overline{\{x\}} \subseteq \overline{\left\{x^{\prime}\right\}}$. A maximal point of $X$ is the generic point of an irreducible component of $X$. If $X$ is quasicompact, for each $x \in X$, there is some closed point $m$, such that $m \leq x$ because the set of all closed subsets is inductive for the relation $\supseteq$ $[4,0.2 .1 .2]$. 
Definition 1 (see [3, Definition 2.2]). Let $f: X \rightarrow Y$ be a morphism of schemes. Then $f$ is called subtrusive if the following two conditions hold.

(1) Every ordered pair $y \leq y^{\prime}$ of points in $Y$ lifts to an ordered pair of points $x \leq x^{\prime}$ in $X$.

(2) $f$ is submersive in the constructible topology.

Then $f$ is called universally subtrusive if $X \times_{Y} Y^{\prime} \rightarrow Y^{\prime}$ is subtrusive for each morphism of schemes $Y^{\prime} \rightarrow Y$.

Clearly, a subtrusive morphism is surjective.

We will mainly consider quasicompact morphisms of schemes. In that case, a morphism of schemes is subtrusive if and only if the above condition (1) holds [3, Proposition 1.6]. As quasicompactness is a universal property, a quasicompact morphism $f$ is universally subtrusive if the condition (1) universally holds for $f$.

Note that a quasicompact morphism of schemes $f: X \rightarrow$ $Y$ is closed if and only if $f$ is specializing; that is, $f(E)$ is stable under specializations for each subset $E \subseteq X$, stable under specializations. To see this use [4, I. Proposition 7.2.12(iv)] which tells us that if $f$ is quasicompact, then $f$ is closed for the patch topology and [4, I, Corollaire 7.3.2] which states that $\bar{F}=\cup[\overline{\{x\}} ; x \in F]$ for a proconstructible subset $F$.

Example 2 (see [3, Remark 2.5]). Let $f: X \rightarrow Y$ be a quasicompact surjective morphism of schemes. Then $f$ is universally subtrusive in the following cases.

(1) $f$ is universally specializing (closed).

(2) $f$ is proper.

(3) $f$ is integral.

(4) $f$ is universally generalizing.

(5) $f$ is faithfully flat.

(6) $f$ is universally open.

Our main results are Artin-Tate-like results, about the descent of the finite type property of morphisms by universally subtrusive morphisms of finite presentation. Artin-Tate's result may be read as follows and exhibits a solution to the 14th problem of Hilbert.

Proposition 3 (see [5, Lemma 3.14.1]). Let $f: X \rightarrow Y$ be a finite and surjective morphism of schemes over a locally Noetherian scheme $S$. Then $X$ is (locally) of finite type over $S$ if and only if $Y$ is (locally) of finite type.

The affine version of this result is quite easy to establish, once the Eakin-Nagata theorem is known. Let $R \rightarrow A \rightarrow B$ be a composite of ring morphisms, such that $R \rightarrow B$ is of finite type, $B$ is Noetherian, and $A \rightarrow B$ is injective integral (equivalently, finite), then $R \rightarrow A$ is of finite type and $A$ is Noetherian. To see this, let $\left\{b_{1}, \ldots, b_{n}\right\}$ be a system of generators of $B$ over $R$ and let $C \subseteq A$ be the $R$-algebra generated by the coefficients of unitary polynomials $p_{i}(X) \in$ $A[X]$, such that $p_{i}\left(b_{i}\right)=0$. Then $C \rightarrow B$ is injective and finite, so that $C$ is Noetherian by the Eakin-Nagata theorem.
It follows that $C \rightarrow A$ is finite and the proof is complete. In passing we note that the Eakin-Nagata theorem is not valid for integral extensions by $[6,2.3]$ : there exists a nonNoetherian domain $R$ such that $R_{P}$ is Noetherian for every prime ideal $P$ of $R$ and such that there exists an integral extension $R \hookrightarrow R^{\prime}$, where $R^{\prime}$ is a Noetherian domain.

The results offered are consequences of a result of Rydh about the structure of universally subtrusive morphisms of finite presentation. Among a lot of nice results, Rydh proved the following.

Theorem 4 (see [3, Theorem 3.10]). Let $Y$ be an affine or Noetherian scheme. Let $f: X \rightarrow Y$ be a universally subtrusive morphism of finite presentation. Then there is a refinement $f^{\prime}: X^{\prime} \rightarrow Y$ of $f$ and a factorization of $f^{\prime}$ into a faithfully flat morphism $X^{\prime} \rightarrow Y^{\prime}$ of finite presentation followed by a proper surjective morphism $Y^{\prime} \rightarrow Y$ of finite presentation. If in addition $f$ is universally open, then one may choose $f^{\prime}$ such that $X^{\prime} \rightarrow X \times{ }_{Y} Y^{\prime}$ is a nil-immersion.

Theorem 4 allows us to reduce our study to proper surjective morphisms. It may be asked whether there is a ring-theoretic version of the preceding result. The answer is no, as the following personal communication of Rydh shows. As a consequence, we cannot reduce the proof to finite morphisms, at least if we wish to use Rydh's above result.

Example 5. Let $Y$ be the affine plane and let $Z \rightarrow Y$ be the blow-up in a point $y$, which is proper. Choose an affine covering $X=Z_{1} \bigsqcup Z_{2}$ of $Z$ and let $f: X \rightarrow Y$ be the natural map. Then $f$ is affine and universally subtrusive but does not admit a refinement of the form $f^{\prime}: X^{\prime} \rightarrow Y^{\prime} \rightarrow Y$ where the first map is faithfully flat and the second is finite and surjective. Indeed, let $I$ be the ideal sheaf defining the point $y$. Since $f^{\prime}$ factors as $X^{\prime} \rightarrow X \rightarrow Z \rightarrow Y$, the ideal sheaf $I O_{X^{\prime}}$ is principal. Since $X^{\prime} \rightarrow Y^{\prime}$ is faithfully flat, this means that the ideal sheaf $I O_{Y^{\prime}}$ is principal. But as $Y^{\prime} \rightarrow Y$ is finite, the inverse image of $y$ has codimension 2 and we have a contradiction.

Thus even in the ring context, we cannot provide a ring theoretic proof of the main theorem of this paper and have to consider morphisms of schemes.

The following results [2, Proposition 16] were extended to schemes by Rydh in [3, Proposition 2.7, Theorem 2.8].

Proposition 6. Let $V$ be a valuation ring and $f: X \rightarrow$ $\operatorname{Spec}(V)$ a morphism of schemes. The following statements are equivalent.

(1) $f$ is universally subtrusive.

(2) $f$ is subtrusive.

(3) The pair $m \leq(0)$ in $\operatorname{Spec}(V)$ lifts to $x \leq x_{0}$ in $X$.

(4) Any chain of points in $\operatorname{Spec}(V)$ lifts to a chain of points in $X$.

(5) There is a closed subscheme $Z \subseteq X$ such that $f_{\mid Z}$ is faithfully flat. 
It follows that a universally subtrusive morphism $f$ : $X \rightarrow Y$ lifts to $X$ chains of points of $Y$ [3, Proposition 2.11], a generalization to schemes of [7, Theorem 3.26]. Actually, the statement of [3, Proposition 2.11] is established for chains that have a lower bound in $Y$, a superfluous condition. To see this, let $Z$ be a subset of a scheme $X$ such that each pair of elements of $Z$ has a lower bound in $Z$. Then the closure of $Z$ is irreducible, and whence has a generic element.

Then we have the following valuative criterion.

Theorem 7. Let $f: X \rightarrow Y$ be a quasicompact morphism.

(1) $f$ is universally subtrusive (resp., submersive) if and only if, for any valuation ring $V$ and morphism $Y^{\prime} \rightarrow$ $Y$ with $Y^{\prime}:=\operatorname{Spec}(V)$, the pull-back $f^{\prime}: X^{\prime} \rightarrow Y^{\prime}$ is subtrusive (resp., submersive).

(2) If $Y$ is locally Noetherian, then it is enough to consider discrete valuation rings in (1), and $f$ is universally subtrusive if and only if $f$ is universally submersive.

Corollary 8. Let $f: X \rightarrow Y$ be a quasicompact morphism of schemes. Then the following statements are equivalent.

(1) $f$ is universally subtrusive.

(2) For every valuation ring $V$ and diagram of solid arrows

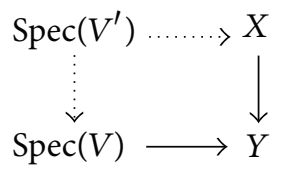

there is a valuation ring $V^{\prime}$ and morphisms such that the diagram becomes commutative and such that the left vertical morphism is surjective.

The above condition (2) is the Nagata's definition of a strongly submersive morphism $[8,9]$. He proved our main theorem for a $B$-algebra, where $B$ is a Nagata ring (pseudogeometric for Nagata).

In order to ease reading, we introduce the following definition.

Definition 9. Let $f: X \rightarrow Y$ be a morphism of schemes. We say that $f$ descends the property $\mathscr{A}$ (resp., property $\mathscr{P}$ ) if for each morphism $g: Y \rightarrow S$, such that $g \circ f$ is of finite type (resp., of finite presentation), then $g$ is of finite type (resp., of finite presentation). We say that $f$ belongs to the class $\mathfrak{A}$ (resp., $\mathfrak{P}$ ) if $f$ descends $\mathscr{A}$ and is of finite type (resp., descends $\mathscr{P}$ and is of finite presentation).

Now we give some comments about the terminology used in this paper. In the literature, a morphism of schemes $X \rightarrow$ $S$ is usually said to descend a property $Q$ of morphisms of schemes if for each morphism $Y \rightarrow S$ such that $Y \times_{S} X \rightarrow X$ has $Q$, then $Y \rightarrow S$ has $Q$. In order to avoid confusions in this paper, we say that such a morphism $X \rightarrow S$ D-descends the property Q (D for diagram).
Remark 10. (1) In case $g \circ f$ is of finite type and $g$ is either quasiseparated or $X$ is Noetherian, then $f$ is of finite type (see [4, I, Proposition 6.3.4]).

(2) If $f$ descends $\mathscr{P}, g \circ f$ is of finite presentation and $g$ is quasiseparated, then $f$ is of finite presentation (see $[4, \mathrm{I}$, Proposition 6.3.8]).

(3) The classes $\mathfrak{A}$ and $\mathfrak{P}$ are stable under right division and composition. In particular, if $f: A \rightarrow B$ is a ring morphism, $A \rightarrow A / I$ descends $\mathscr{A}(\mathscr{P})$ for each ideal $I \subseteq$ ker $f$ of $A$ if $f$ does.

(4) We examine a converse. Suppose that $A / \operatorname{ker} f \rightarrow B$ descends $\mathscr{A}$ and that ker $f$ is a nilpotent ideal. Then $A \rightarrow$ $B$ descends $\mathscr{A}$ by $[10$, Lemma 4$]$. The nilpotent condition on ker $f$ is verified in two cases.

(a) If $A \rightarrow B$ is of finite presentation, surjective on the spectra and $A \rightarrow A / \operatorname{ker} f$ descends the property $\mathscr{P}$, then $\operatorname{ker} f$ is a nilpotent ideal, because $\sqrt{\operatorname{ker} f}=$ $\sqrt{0}$ and ker $f$ is an ideal of $A$ of finite type by [4, I, Remarque 6.2.7.2], since $A \rightarrow A / \operatorname{ker} f$ is of finite presentation.

(b) A ring morphism $f: A \rightarrow B$ is called a strong Nakayama morphism if $M \otimes_{A} B \neq 0$ for each nonzero $A$-module $M$. A strong Nakayama morphism Ddescends the finite type and finite properties of ring morphisms [11, Proposition 2.6, page 30]. Morphisms $f$ that $\mathrm{D}$-descend the finite presentation property of ring morphisms are Nakayama morphisms such that ker $f$ is a Noetherian $A$-module contained in $\sqrt{0}$, by the two lemmas of [11, page 31] and since $A \rightarrow$ $A /$ ker $f \mathrm{D}$-descends the finite presentation property.

(c) Suppose that a morphism $X \rightarrow S$ of schemes universally descends the property $\mathscr{A}$ and is of finite type, then $X \rightarrow S$ universally $\mathrm{D}$-descends the property $\mathscr{A}$. To see this consider the pull-back defined by the maps $X \rightarrow S$ and $Y \rightarrow S$. An example is given by a faithfully flat morphism of finite presentation by Theorem 11.

Some results about immersions and monomorphisms are involved. They will be recalled when needed, especially in Section 2. Section 6 is concerned with topological immersions of schemes, a notion "dual" of submersions. They are considered because immersions of schemes are topological immersions. Moreover, the results we get have their own interest. A morphism of schemes $f: X \rightarrow Y$ is called a topological immersion if $f$ is injective and if the topology of $X$ coincides with the inverse image topology on $X$, with respect to $f$. In case $f$ is quasicompact, the topological immersion property can be characterized with ordered pair of points of $X$, similarly to the definition of subtrusive morphisms. We also consider topological essentiality, which in the affine case is linked to essential morphisms of rings.

Section 2 deals with faithfully flat morphisms of schemes, of finite presentation, that are known to descend $\mathscr{A}$ and $\mathscr{P}$. We derive some results from this case. In particular, we prove our main result for universally subtrusive $S$-morphisms of schemes $X \rightarrow \operatorname{Spec}(V)$, where $V$ is a valuation domain. 
In the affine context, the hypothesis that $V$ is a valuation domain can be replaced by $V$ is a Prüfer domain. As an easy consequence of the theorem of generic flatness for a surjective $S$-morphism of schemes $X \rightarrow Y$, where $Y$ is concentrated and reduced, we get that if $X \rightarrow S$ is of finite type (of finite presentation), there is a dense open subset $O$ of $Y$, such that $O \rightarrow Y \rightarrow S$ is of finite type (of finite presentation). This will be useful in Section 3, where the Noetherian case is studied. We introduce absolutely flat schemes and recall their main properties, in order to get "essentially" Artin-Tate-like results. Absolutely flat schemes are also used in Section 4 . For instance, consider ring morphisms $S \rightarrow A \rightarrow B$, where $S \rightarrow$ $B$ is of finite type and $A \rightarrow B$ is of finite type and spectrally surjective. Then for each finite type point (Goldman point) $P$ of $A$ (i.e., $A \rightarrow \kappa(P)$ is of finite type), $S \rightarrow A \rightarrow \kappa(P)$ is of finite type. In particular, $S \rightarrow A / M$ is of finite type for each maximal ideal $M$ of $A$. This is established in the scheme context.

In Section 3, we consider the Noetherian case and exhibit an Artin-Tate-like result. A result established by Onoda is crucial: if $R$ is a Noetherian domain and $A$ an overdomain of $R$, then the ring morphism $R \rightarrow A$ is of finite type provided that $R_{P} \rightarrow A_{P}$ is of finite type for each $P \in \operatorname{Spec}(A)$ and there is a nonzero element $x$ of $A$ such that $R \rightarrow A_{x}$ is of finite type. The second condition is gotten as a consequence of the Theorem of generic flatness. The local condition is deduced by faithfully flat descent from a result by Hashimoto, when the base ring is excellent.

Section 4 examines properties of universally closed morphisms of schemes. We thank Bjorn Poonen for his kind authorization to reproduce his proof of the following result, electronically published in [12]. A universally closed morphism is quasicompact. A version of the proof also appears in the Stacks Project [13, Lemma 39.9]. Within the category of affine schemes, universally closed morphisms and integral morphisms coincide. Recall that a morphism of schemes $f$ : $X \rightarrow Y$ is called a Stein morphism if $\mathcal{O}_{Y} \rightarrow f_{\star} \mathcal{O}_{X}$ is an isomorphism. We give Stein factorization results published in [13] for concentrated universally closed morphisms $f$ : $X \rightarrow S$. In that case, there is a factorization $X \rightarrow$ $S^{\prime} \rightarrow S$, where the first morphism is Stein and the second is integral. We define incomparable morphism of schemes in the same way as in Commutative Algebra. If a universally closed (proper) separated morphism $f$ is incomparable, then $f$ is integral (finite). As a consequence, we give a short proof of a Raynaud's result: if $g \circ f$ is integral, $f$ surjective, and $g$ separated, then $g$ is integral.

In Section 5, we introduce pure morphisms of schemes defined and characterized by Mesablishvili, an extension to schemes of pure morphisms of rings. We consider only the quasicompact context, in which case pure morphisms are nothing but universally schematically dominant morphisms of schemes. We show that they are universally subtrusive when concentrated. Moreover, quasicompact universally subtrusive morphisms are shown to be quasicompact morphisms that become pure after each base change with respect to a valuation domain. We also establish a criterion for a flat morphism to be schematically dominant by using the set of all (weak Bourbaki) associated primes of a scheme.
Section 6 is concerned with monomorphisms of schemes and (topological) immersions. We are mainly interested in quasicompact flat monomorphisms. We look at relations with strict monomorphisms and quasiaffine morphisms. For a quasicompact morphism, the topological immersion property is equivalent to some property of pairs of comparable elements of schemes. We define topologically essential and schematically essential morphisms of schemes. We examine their properties linked to topologically minimal continuous maps.

\section{Faithfully Flat Morphisms}

Considering Theorem 4, we see that faithfully flat morphisms of finite presentation are involved. In this case the main theorem is already known.

Theorem 11 (see [14-17, Proposition 11.3.16]). A faithfully flat morphism of schemes of finite presentation descends $\mathscr{A}$ and $\mathscr{P}$.

We next derive some consequences of this result. Some authors say that a scheme (resp., a morphism) is concentrated if it is quasicompact and quasiseparated. Let $f: X \rightarrow$ $Y$ be a morphism of schemes, such that $Y$ is concentrated. Then $X$ is concentrated if and only if $f$ is concentrated (see [4, I, Section 6] for proofs). Noetherian schemes and affine schemes are concentrated. Note that a quasicompact $U$ subset of a concentrated scheme $X$ is such that $U \rightarrow$ $X$ is quasicompact because $X$ is quasiseparated. Actually, if $X$ is concentrated, $U \rightarrow X$ is concentrated for each quasicompact open subset $U$ of $X$, and so is $U$. For a concentrated morphism of schemes, the finite type (finite presentation) property is equivalent to the locally finite type (locally finite presentation) property by the very definition of these properties.

We need some considerations about monomorphisms of schemes. Note that monomorphisms between affine schemes correspond to epimorphisms of the category of commutative rings.

Proposition 12 (see [18, Proposition 1.1]). Let $f: X \rightarrow Y$ be a scheme morphism. The following statements are equivalent.

(1) $f$ is a flat monomorphism.

(2) $f$ is injective and $\mathcal{O}_{Y, f(x)} \rightarrow \mathcal{O}_{X, x}$ is an isomorphism for each $x \in X$.

Proposition 13. Properties of monomorphisms are as follows.

(1) A monomorphism is separated because its diagonal morphism is an isomorphism.

(2) in [19, page 100], an immersion is a monomorphism. A quasicompact flat monomorphism $f$ is an isomorphism if and only if $f$ is surjective. A finite monomorphism is a closed immersion.

(3) in [14-17, Proposition 17.9.1], an open immersion $f$ is a flat monomorphism locally of finite presentation. In case $f$ is quasicompact, then $f$ is of finite presentation. 
Let $X$ be a scheme and $x \in X$, then the natural map $\mathcal{O}_{X, x} \rightarrow X$ is a flat monomorphism with image $\mathrm{g}(x)$, the set of all generalizations of $x$.

The following lemma is useful to reduce proofs to affine schemes.

Lemma 14. Let $X$ be a concentrated scheme and $\left\{U_{1}, \ldots, U_{n}\right\}$ be an affine open covering of $X$. Set $Z:=\bigsqcup_{i=1, \ldots, n} U_{i}$, the following statements hold.

(1) Each morphism $U_{i} \rightarrow X$ is concentrated and a flat monomorphism of finite presentation.

(2) The canonical morphism $g: Z \rightarrow X$ is concentrated, of finite presentation, faithfully flat and $Z$ is an affine scheme.

Proof. (1) We can use the above remark on concentrated schemes. We give a direct proof. Each $U_{i} \rightarrow X$ is an open immersion, whence a flat monomorphism locally of finite presentation by Proposition 13. Moreover, $U_{i}$ is proconstructible, whence retrocompact $[4, \mathrm{I}$, Proposition 7.2.3(ix),(v)]. It follows that $U_{i} \rightarrow X$ is quasicompact and therefore of finite presentation.

(2) Clearly, $g$ is faithfully flat and of finite presentation by [4, I, Proposition 6.3.11]. Now $g$ is concentrated because of [4, I, Proposition 6.1.15].

Proposition 15. Let $V$ be a valuation domain, $S \rightarrow V$ a ring morphism, $X$ a quasiseparated scheme, and $f: X \rightarrow \operatorname{Spec}(V)$ a universally subtrusive morphism of schemes of finite type, such that $X \rightarrow \operatorname{Spec}(V) \rightarrow \operatorname{Spec}(S)$ is of finite type (of finite presentation). Then $S \rightarrow V$ is of finite type (of finite presentation).

Proof. In view of [3, Proposition 2.7(vii)], there is a closed subscheme $Z$ of $X$ such that $Z \rightarrow X \rightarrow \operatorname{Spec}(V)$ is faithfully flat. Then $Z \rightarrow X$ is of finite type and so is $Z \rightarrow$ $\operatorname{Spec}(V)$. Moreover, a closed immersion is quasicompact and separated, whence $Z \rightarrow X \rightarrow \operatorname{Spec}(V)$ is concentrated because $f$ is concentrated. Since $Z$ is concentrated, we can suppose that $Z$ is affine by Lemma 14 . We are now in position to apply [20, I, Corollaire 3.4.7] which tells us that a flat ring morphism of finite type, whose domain is an integral domain, is of finite presentation. Then $Z \rightarrow \operatorname{Spec}(V)$ is faithfully flat of finite presentation and therefore $S \rightarrow V$ is of finite type (resp., of finite presentation) by Theorem 11.

In the affine context, we can give a simpler proof. We introduce the following definition.

Definition 16. A universally injective ring morphism is called pure. A pure ring morphism is universally subtrusive [2, page 535]. A faithfully flat ring morphism is pure.

Results about pure ring morphisms used in this paper come from the work of Olivier [11]. Pure morphisms of schemes are introduced in Section 5.

Proposition 17. Let $A$ be a Prüfer domain, $S \rightarrow A$ a ring morphism, and $f: A \rightarrow B$ a universally subtrusive ring morphism of finite type, such that $S \rightarrow B$ is of finite type, then so is $S \rightarrow A$.

Proof. Since each $A_{P}$ for $P \in \operatorname{Spec}(A)$ is a valuation domain, we get that $A_{P} \rightarrow B_{p}$ is pure by [2, Théorème 37(a)] and then $A \rightarrow B$ is pure. Let $K$ be the quotient field of $A$ and $I:=\operatorname{ker}\left(B \rightarrow B \otimes_{A} K\right)$ the torsion ideal of $f$. Then by [11, Corollaire 1.5], $A \rightarrow B / I$ is pure and flat because $A$ is Prüfer and $A \rightarrow B / I$ is torsion-free. Therefore, $A \rightarrow B / I$ is faithfully flat and $A \rightarrow B / I$ of finite type. It follows from [20, I, Corollaire 3.4.7] that $A \rightarrow B / I$ is of finite presentation and $S \rightarrow B / I$ of finite type. Therefore, $S \rightarrow A$ is of finite type.

The following result is well known. A proof may be found in the Columbia Stack project [13, Proposition 25.2] included in a stronger result.

Proposition 18 (Theorem of generic flatness). Let $f: X \rightarrow$ $Y$ be a morphism of schemes of finite type, such that $Y$ is reduced. Then there exists a dense open subscheme $U$ of $Y$ such that $X_{U}:=X \times_{Y} U \rightarrow U$ is flat and of finite presentation.

Corollary 19. Let $f: X \rightarrow Y$ be an S-morphism of schemes of finite type, surjective, and such that $Y$ is reduced and concentrated. If $X \rightarrow S$ is of finite type (resp., of finite presentation), there is a nonempty affine open subset $O$ of $Y$ such that $O \rightarrow Y \rightarrow S$ is of finite type (resp., of finite presentation).

In case $S=\operatorname{Spec}(A)$ and $Y=\operatorname{Spec}(B)$ are affine schemes where $Y$ is reduced, one gets that there is some regular element $b \in B$ such that $A \rightarrow B_{b}$ is of finite type (resp., of finite presentation), with $B_{b} \subseteq \operatorname{Tot}(B)$.

Proof. Use the base change $U \rightarrow Y$ defined in Proposition 18, pick some nonempty affine open subset $O \subseteq U$ and observe that $O \rightarrow Y$ is a quasicompact open immersion by Lemma 14, whence of finite presentation and so is $X_{O} \rightarrow$ $X$.

In case $S=\operatorname{Spec}(A)$ and $Y=\operatorname{Spec}(B)$ are affine schemes where $Y$ is reduced in Proposition 18, we see that $U=\mathrm{D}(I)$ is such that $0: I=0$; that is, $I$ is dense.

We recall that a scheme is called absolutely flat if each of its stalks is a field. Olivier and Hochster proved independently in $[21,22]$ the existence of a universal absolutely flat scheme $X^{\text {cons }}($ or $T(X)$ ) associated with an arbitrary scheme $X$, a construction announced by Grothendieck in [4, I, 7.2.14]. Actually, $T(X)$ is the final object of the category of absolutely flat $X$-schemes. The structural morphism $t_{X}: T(X) \rightarrow X$ is an affine surjective monomorphism, and the canonical map $t_{X}: T(X) \rightarrow X$ is a homeomorphism when $X$ is endowed with the constructible topology. In particular for a ring $A$, there is a ring epimorphism $A \rightarrow T(A)$ which gives for $S=\operatorname{Spec}(A)$, the structural morphism $T(S) \rightarrow S$. Since an affine morphism is quasicompact and separated, we see that $t_{X}$ is concentrated for an arbitrary scheme $X$. 
Proposition 20 (see [23, Lemma 8.4, Proposition 8.5]). Let $\left(X, \mathcal{O}_{X}\right)$ be a ringed space, such that $\mathcal{O}_{X, x}$ is a field for each $x \in X$.

(1) $\mathcal{O}_{X}(X)$ is absolutely flat.

(2) $X$ is an affine scheme if and only if $X$ is a concentrated scheme. If the preceding condition is verified, then $X$ is an absolutely flat scheme.

Corollary 21. Let $X$ be a concentrated scheme, then $T(X)$ is an affine scheme.

Proposition 22 (see [23, Lemme 8.6]). Let $f: X \rightarrow S$ be a quasicompact monomorphism of schemes. If $S$ is absolutely flat, then $f$ is a flat closed immersion.

Proposition 23. Let $X \rightarrow Y \rightarrow S$ be scheme morphisms and $Y^{\prime} \rightarrow Y$ a scheme morphism of finite type, where $Y^{\prime}$ is absolutely flat. If $X \rightarrow Y$ is surjective and of finite presentation and $X \rightarrow S$ of finite type, then $Y^{\prime} \rightarrow Y \rightarrow S$ is of finite type.

Proof. Observe that $X \times_{Y} Y^{\prime} \rightarrow Y^{\prime}$ is faithfully flat of finite presentation and that $X \times_{Y} Y^{\prime} \rightarrow S$ is of finite type.

We intend to apply the above result in order to obtain "almost" Artin-Tate-like results.

Remark 24. Let $A$ be a reduced $G$-ring (that is, $A \rightarrow \operatorname{Tot}(A)$ is of finite type or, equivalently $\operatorname{Tot}(A)=A_{s}$ for some regular $s \in A$ ) and suppose that $\operatorname{Tot}(A)$ is absolutely flat, then for a composite of ring morphisms which is of finite type $S \rightarrow$ $A \rightarrow B$, with $f: A \rightarrow B$ of finite presentation and such that ${ }^{a} f$ is surjective, then $S \rightarrow A \rightarrow \operatorname{Tot}(A)$ is of finite type because $\operatorname{Tot}(A) \rightarrow \operatorname{Tot}(A) \otimes_{A} B$ is faithfully flat and of finite presentation. In particular, if $A$ is a $G$-domain, we get that there is some nonzero $f \in A$ such that $S \rightarrow A_{f}$ is of finite type.

Note that a reduced ring $A$ is such that $\operatorname{Tot}(A)$ is absolutely flat if and only if $\operatorname{Min}(A)$ is compact (Hausdorff) and $A$ is a McCoy ring; that is, each finitely generated ideal contained in $Z(A)$ has a nonzero annihilator. Such rings are called decent in [24, page 259]. In particular a ring with few zero divisors is decent, like a Noetherian reduced ring.

The above letter $G$ refers to some Goldman property of a ring. We can also define Goldman points of a scheme $S$. A finite type point $s$ of $S$ is a point such that $\operatorname{Spec}(\kappa(s)) \rightarrow$ $\operatorname{Spec}\left(\mathcal{O}_{S, s}\right) \rightarrow S$ is of finite type (see [13, Section 15]). In Commutative Algebra, such points are called Goldman points, a terminology we keep. A point $s \in S$ is Goldman if and only if $\{s\}$ is a locally closed subset of $S$, that is, of the form $\overline{\{x\}} \cap U$, where $U$ is an affine open subset. We denote by $\operatorname{Gold}(X)$ the set of all Goldman points of $X$. Observe that $\operatorname{Gold}(X)$ is strongly dense in $X$ by [13, Lemma 15.6] and [4, Section 0.2.6]. It contains the set of all closed points and the set of all isolated points, the so-called $g$-points.

Proposition 25. Let $f: X \rightarrow S$ be a surjective morphism of schemes of finite type and $g: S \rightarrow T$ a morphism of schemes such that $g \circ f$ is of finite type (resp., of finite presentation). Let $s \in \operatorname{Gold}(S)$, then $\operatorname{Spec}(\kappa(s)) \rightarrow S \rightarrow T$ is of finite type (resp., of finite presentation).

Proof. Similar to the proof of Remark 24.

We can factorize $\operatorname{Spec}(\kappa(s)) \rightarrow S$ as $\operatorname{Spec}(\kappa(s)) \rightarrow$ $\operatorname{Spec}\left(\mathcal{O}_{S, s}\right) \rightarrow S$ if $s \in S$, where the first morphism is a closed immersion and the second is a quasicompact flat monomorphism. In case $\operatorname{Spec}\left(\mathcal{O}_{S, s}\right)$ is Artinian and the above hypotheses hold, $\operatorname{Spec}\left(\mathcal{O}_{S, s}\right) \rightarrow S \rightarrow T$ is of finite type, by [13, Lemma 15.2].

Corollary 26. Let $f: X \rightarrow \operatorname{Spec}(B)$ be a surjective morphism of schemes of finite type and $\varphi: A \rightarrow B$ a ring morphism, such that $X \rightarrow \operatorname{Spec}(A)$ is of finite type (resp., of finite presentation). Then $A \rightarrow B \rightarrow B / M$ is of finite type (resp., of finite presentation) for each maximal ideal $M$ of $B$.

In view of [13, Lemma 15.2], if $A \rightarrow B \rightarrow B / M$ is of finite type and $(B, M)$ is Artinian local, then $A \rightarrow B$ is of finite type.

Remark 27. Note that if $S$ is a subring of $A$ and $S\left[X_{1}, \ldots, X_{n}\right]=A\left[X_{1}, \ldots, X_{n}\right]$, then $S \rightarrow A$ is of finite presentation because $A \rightarrow A\left[X_{1}, \ldots, X_{n}\right]$ is faithfully flat of finite presentation. This is an application to the nonunicity of the coefficient ring of a polynomial ring.

\section{The Noetherian Context}

We will prove an Artin-Tate-like result under Noetherian hypotheses. We need a result proved by Onoda [25, Lemma 2.14, Theorem 2.20] in a more general setting.

Theorem 28. Let $R$ be a Noetherian domain and $A$ an overdomain of $R$ such that $A_{x}$ is finitely generated over $R$ for some nonzero $x \in A$. Then the following statements hold.

(1) If $S$ is a multiplicative subset of $R$ such that $R_{S} \rightarrow A_{S}$ verifies $\mathscr{A}$, then there is some $s \in S$ such that $R \rightarrow A_{s}$ verifies $\mathscr{A}$.

(2) $R \rightarrow A$ verifies $\mathscr{A}$ if and only if $R_{M} \rightarrow A_{M}$ verifies $\mathscr{A}$ for each maximal ideal (resp., prime ideal) $M$ of $R$.

Corollary 29. Let $R$ be a Noetherian domain and $A$ an $R$ subalgebra of a finitely generated overdomain of $R$. Then the statements (1) and (2) of Theorem 28 hold.

Proof. In view of [26, Proposition 2.1(b)], there is some $f \in A$ such that $R \rightarrow B_{f}$ is of finite type.

Another results proved by Fogarty will be useful [27, page 169]. They are developed by Alper in unpublished notes [28, Lemma 3.1].

Lemma 30. Let $A \rightarrow B$ be a ring morphism between Noetherian rings.

(1) $B$ is finitely generated over $A$ if and only if $B_{\text {red }}$ is finitely generated over $A$. 
(2) Let $\operatorname{Spec}(B)=\cup_{i} \operatorname{Spec} B_{i}$ be the irreducible decomposition. Then $B$ is finitely generated over $A$ if and only if each $B_{i}$ is finitely generated over $A$.

Next result is decisive.

Proposition 31 (see [29, Theorem 4.2]). Let $S$ be an excellent ring, and $f: X \rightarrow Y$ a surjective proper morphism of $S$ schemes. If $X$ is of finite type over $S$ and $Y$ is a Noetherian affine scheme, then $Y$ is of finite type over $S$.

It deserves to be compared with the following Alper's result.

Proposition 32 (see [28, Proposition 1.3]). Let $R$ be an excellent ring and $f: X \rightarrow Y$ a morphism of $R$-schemes. Suppose the following:

(1) $f$ is surjective.

(2) $X$ is of finite type over $R$.

(3) Each irreducible component of $X$ dominates $Y$.

(4) $Y$ is normal and Noetherian.

Then $Y$ is of finite type over $R$.

We recall the following descent result by base changes.

Proposition 33 (see [11, Proposition 5.3, page 22]). Let $f$ : $A \rightarrow B$ be a ring morphism. Then $f$ is pure if and only if $f$ universally $D$-descends the property $\mathscr{P}$.

Corollary 34. Let $S$ be a Noetherian ring and $f: X \rightarrow Y a$ surjective proper morphism of $S$-schemes, where $Y:=\operatorname{Spec}(R)$ is an affine Noetherian scheme. If $X$ is of finite type over $S$, so is $S_{P} \rightarrow R_{P}$ for each prime ideal $P$ of $S$.

Proof. For each $P \in \operatorname{Spec}(S)$, consider the base change $S \rightarrow$ $S_{P} \rightarrow \widehat{S_{P}}$, where $\widehat{S_{P}}$ is the $P S_{P}$-adic completion of the local ring $S_{P}$ and is excellent. It follows by Proposition 31 and the descent of the finite type property by the faithfully flat morphism $S_{P} \rightarrow \widehat{S_{P}}$ that $S_{P} \rightarrow R_{P}$ is of finite type.

We can now state the main result of the section.

Theorem 35. Let $f: X \rightarrow Y$ be a universally subtrusive morphism of S-schemes and suppose that $Y$ and $S$ are Noetherian. If $X \rightarrow S$ is of finite type, so is $Y \rightarrow S$.

Proof. First observe that $X \rightarrow Y$ is of finite presentation because of finite type and $Y$ is Noetherian [4, I, 6.3]. In view of [3, Theorem 3.10] there is a morphism of schemes $X^{\prime} \rightarrow X$ such that $f^{\prime}: X^{\prime} \rightarrow Y$ can be factored into a faithfully flat morphism of finite presentation $X^{\prime} \rightarrow Y^{\prime}$ followed by a proper surjective morphism $Y^{\prime} \rightarrow Y$. Clearly, $X^{\prime} \rightarrow S$ is of finite type and by Theorem 11, $Y^{\prime} \rightarrow S$ is of finite type. Therefore, we can assume that $X \rightarrow Y$ is a proper surjective morphism. We can also assume that $S:=\operatorname{Spec}(R)$ and $Y:=\operatorname{Spec}(B)$ are affine schemes with a ring morphism $\varphi$ : $R \rightarrow B$. Using the base changes $B \rightarrow B_{\text {red }}$ and $B \rightarrow B / M$ for each minimal prime ideal $M$ of $B$ for $X \rightarrow Y$, we can assume that $B$ is integral by [27, page 169] or by Lemma 30(2). Furthermore, we can suppose that $R$ is integral by changing $R$ with $R / \operatorname{ker} \varphi$ because $X \rightarrow Y \rightarrow \operatorname{Spec}(R / \operatorname{ker} \varphi)$ is of finite type. The proof is completed by combining Theorem 28, Corollaries 29 and 19.

Remark 36. Let $f: A \rightarrow B$ be a finite ring morphism of $S$ algebras such that the canonical map $\operatorname{Spec}(B) \rightarrow \operatorname{Spec}(A)$ is surjective and suppose that $S \rightarrow B$ verifies $\mathscr{A}$. In view of [30, Corollary 1] there is some affine $S$-subalgebra $A^{\prime}$ of $A$, such that $A^{\prime} \rightarrow B$ is a finite morphism and the module generators of $B$ over $A$ and $A^{\prime}$ are the same. To see this it is enough to reduce the proof to an injective ring morphism $A \rightarrow B$.

Remark 37. Some known results are not a consequence of Theorem 35 and Proposition 17. They concern the so-called strongly affine pairs of rings $A \subseteq B$ (such that $A$ is a subring of $B$ and such that each $A$-subalgebra of $B$ is of finite type). For instance, if $A$ is a field and $B=A[x]$ an $A$-algebra with a single generator, $A \subseteq B$ is a strongly affine pair [31]. The reader will find much more examples in a paper by Papick [32]. Note also that in case $A \subseteq C$ is a pair of rings sharing an ideal $I$, then an $A$-subalgebra $B$ of $C$ is of finite type if and only if so is $A / I \rightarrow B / I$.

\section{Universally Closed Morphisms}

In this section, we give a survey about new results on universally closed morphisms and add some commentaries.

We thank Bjorn Poonen for his kind authorization to reproduce his proof of the next result, published electronically in [12]. A version of the solution appears also in the Stacks Project [13, Lemma 21.39.9].

Theorem 38. A universally closed morphism of schemes $f$ : $X \rightarrow S$ is quasicompact. If, in addition, $f$ is also surjective, then it is universally subtrusive.

Proof. Without loss of generality, we may assume that $S=$ $\operatorname{Spec}(A)$ for some ring $A$ and that $f$ is surjective. Suppose that $f$ is not quasicompact. We need to show that $f$ is not universally closed.

Write $X=\cup_{i \in I} X_{i}$ where the $X_{i}$ are affine open subschemes of $X$. Let $T:=\operatorname{Spec}\left(A\left[\left\{t_{i}: i \in I\right\}\right]\right)$, where the $t_{i}$ are distinct indeterminates. Let $T_{i}:=\mathrm{D}\left(t_{i}\right) \subseteq T$. Let $Z$ be the closed set $\left(X \times_{S} T\right) \backslash \cup_{i \in I}\left(X_{i} \times{ }_{S} T_{i}\right)$. It suffices to prove that the image $f_{T}(Z)$ of $Z$ under $f_{T}: X \times_{S} T \rightarrow T$ is not closed. There exists a point $P \in \operatorname{Spec}(A)$ such that there is no neighborhood $U$ of $P$ in $S$ such that $X_{U}$ is quasicompact, since otherwise we could cover $S$ with finitely many such $U$ and prove that $X$ itself was quasicompact. Fix such $P$, and let $k$ be its residue field. First we check that $f_{T}\left(Z_{k}\right) \neq T_{k}$. Let $\tau \in T(k)$ be the point such that $t_{i}(\tau)=1$ for all $i$. Then $\tau \in T_{i}$ for all $i$, and the fiber of $Z_{k} \rightarrow T_{k}$ above $\tau$ is isomorphic to $\left(X \backslash \cup_{i \in I} X_{i}\right)_{k}$, which is empty. Thus, $\tau \in T_{k} \backslash f_{T}\left(Z_{k}\right)$. If $f_{T}(Z)$ were closed in $T$, there would exist a polynomial $g \in A\left[\left\{t_{i}: i \in I\right\}\right]$ vanishing on $f_{T}(Z)$ but not at $\tau$. Since $g(\tau) \neq 0$, some coefficient of $g$ would have nonzero image in $k$, and hence be invertible on some neighborhood $U$ of $P$. Let $J$ be the finite set of $j \in I$ 
such that $t_{j}$ appears in $g$. Since $X_{U}$ is not quasicompact, we may choose a point $x \in X \backslash \cup_{j \in J} X_{j}$ lying above some $u \in U$. Since $g$ has a coefficient that is invertible on $U$, we can find a point $Q \in T$ lying above $u$ such that $g(Q) \neq 0$ and $t_{i}(Q)=0$ for all $i \notin J$. Then $Q \notin T_{i}$ for each $i \notin J$. A point $z$ of $X \times{ }_{S} T$ mapping to $x \in X$ and to $Q \in T$ then belongs to $Z$. But $g\left(f_{T}(z)\right)=g(Q) \neq 0$, so this contradicts the fact that $g$ vanishes on $f_{T}(Z)$.

If $f$ is surjective and universally closed, then $f$ is universally subtrusive by the first part of the proof and Example 2.

Rydh observed in the same item of [12] that Bjorn Poonen's argument can be somewhat simplified by using the simple (topological) fact that a closed morphism with quasicompact fibers is quasicompact (this is implicit in his argument). In the above proof we can thus assume that $S$ is a point and that $X$ is not quasicompact. Another comment by Rydh is Proposition 41(1), which shows that a separated and universally closed morphism is "almost proper." We will need the following results.

Let $f: X \rightarrow S$ be a concentrated morphism, so that $f_{\star} \mathcal{O}_{X}$ is a quasicoherent $\mathcal{O}_{S}$-algebra. We consider below its relative spectrum $\operatorname{Spec}_{S}\left(f_{*} \mathcal{O}_{X}\right)$ over $S$ (see [4, I, Section 9]).

Proposition 39 (Stein Factorization [33, Lemmas 29.1 and 29.5]). Let $S$ be a scheme. Let $f: X \rightarrow S$ be a universally closed (whence quasicompact) and quasiseparated morphism of schemes. There exists a factorization $\pi \circ f^{\prime}$ of $f$ where $\pi$ : $S^{\prime} \rightarrow S$ and $f^{\prime}: X \rightarrow S^{\prime}$ with the following properties:

(1) the morphism $f^{\prime}$ is universally closed, quasicompact, quasiseparated, and surjective,

(2) the morphism $\pi: S^{\prime} \rightarrow S$ is integral whence affine,

(3) one has $f_{*}^{\prime} \mathcal{O}_{X}=\mathcal{O}_{S^{\prime}}$; that is, $f^{\prime}$ is a Stein morphism,

(4) one has $S^{\prime}=\operatorname{Spec}_{S}\left(f_{*} \mathcal{O}_{X}\right)$,

(5) $S^{\prime}$ is the normalization of $S$ in $X$,

(6) if $X \rightarrow S$ is locally of finite type, then $S^{\prime} \rightarrow S$ is finite.

In case $f$ is proper then $f^{\prime}$ is proper with geometrically connected fibers.

The following result generalizes [34, Proposition 3.18].

Corollary 40. Let $f: X \rightarrow S$ be a universally closed and quasiseparated morphism of schemes, where $S=\operatorname{Spec}(A)$ is an affine scheme. Then $\varphi: A \rightarrow \mathcal{O}_{X}(X)$ is an integral ring morphism.

Proof. By the above proposition, $f$ can be factored $\pi \circ f^{\prime}$, where $\pi$ is integral and $f^{\prime}$ is a Stein morphism. In view of [35, Proposition 6.1.4], $A \rightarrow \mathcal{O}_{S^{\prime}}\left(S^{\prime}\right)$ is integral and then the proof is complete since $\mathcal{O}_{X}(X)=\mathcal{O}_{S^{\prime}}\left(S^{\prime}\right)$.

Proposition 41. Let $f: X \rightarrow$ S be a separated and universally closed morphism of schemes. Let $Y:=X_{s}$ be the fiber of $f$ for some $s \in S, R:=\mathcal{O}_{Y}(Y)$ and $\varphi: Y \rightarrow \operatorname{Spec}(R)$ the canonical morphism.
(1) Y has finite dimension.

(2) $R$ is integral over $\kappa(s)$, whence is zero-dimensional.

(3) $Y$ is concentrated and the canonical map $R_{f} \rightarrow$ $\mathcal{O}_{Y}\left(Y_{f}\right)$ is an isomorphism, where each $\varphi^{-1}(D(f))=$ $Y_{f}$ for $f \in R$ is open and closed.

Proof. (1) It is enough to use [36, Corollary 8.4] because a closed immersion is finite and the diagonal of $f$ is a closed immersion.

(2) Use Corollary 40.

(3) By [4, Corollaire 6.1.10], $Y$ is quasiseparated. The proof of the isomorphism is [37, Lemma 14.2]. Now the reduced ring of $R$ is absolutely flat so that each element of $R_{\text {red }}$ is of the form eu where $e$ is an idempotent and $u$ is a unit. It follows that $X_{f}$ is open and closed because so is $\mathrm{D}(e)$ in $\operatorname{Spec}\left(R_{\text {red }}\right)$.

We have defined a preorder $\leq$ on the underlying set of a scheme $X$ by $x \leq x^{\prime} \Leftrightarrow x \in \overline{\left\{x^{\prime}\right\}}$. A morphism of schemes $f: X \rightarrow Y$ is called incomparable if for any pair $x \leq x^{\prime}$ of elements of $X$, then $f(x)=f\left(x^{\prime}\right) \Rightarrow x=x^{\prime}$, or equivalently, each of the points of $X_{y}$ is maximal for each $y \in Y$; that is, each fiber is zero-dimensional. Clearly, an integral morphism of schemes is incomparable and so is an injective morphism. A composite of incomparable morphisms is incomparable. If $g \circ f$ is incomparable and $f$ is subtrusive, then $g$ is incomparable. Note that the morphism $g$ of Lemma 14 is incomparable.

Olivier defined absolutely flat morphisms as flat morphisms, whose diagonal morphisms are flat [38]. We may find in $[19,(1.4)]$ that a morphism of schemes $Z \rightarrow S$, where $S$ is the spectrum of a field $k$, is absolutely flat if and only if $L_{z}:=\mathcal{O}_{Z, z}$ is a field and $k \rightarrow L_{z}$ is a separable algebraic extension for each $z \in Z$. In this case, $Z$ is an absolutely flat scheme. It follows that an absolutely flat morphism of schemes is universally incomparable. Recall that a morphism is étale if and only if it is absolutely flat and locally of finite presentation.

Proposition 42 (see [39, Corollary 12.90, page 359]). Let $f$ : $X \rightarrow Y$ be a proper morphism of schemes and let $V$ be the set of $y \in Y$ such that $f^{-1}(y)$ is a finite set. Then $V$ is open in $Y$ and the restriction $f^{-1}(V) \rightarrow V$ of $f$ is a finite morphism.

Corollary 43. A proper, incomparable, and surjective morphism of schemes $f: X \rightarrow Y$ is finite.

Proof. Let $y$ be a point of $Y$. Then $X_{y}:=f^{-1}(y)$ is nonempty, quasicompact, and Noetherian and each of its points is closed. By [4, I, Proposition 2.8.1] $X_{y}$ is finite. It follows that $V=Y$.

The equivalence $(1) \Leftrightarrow(2)$ in the following theorem is an answer by Rydh to a question of Grothendieck [14-17, Remarque 18.12.9]. 
Theorem 44. Let $f: X \rightarrow Y$ be a separated morphism of schemes. The following statements are equivalent:

(1) $f$ is integral,

(2) $f$ is universally closed and has affine fibers [36, Theorem 8.5],

(3) $f$ is universally closed and incomparable.

Proof. (1) $\Leftrightarrow$ (3). Clearly an integral morphism verifies (3). Let $f: X \rightarrow Y$ be a separated, universally closed, and incomparable morphism. Let $F$ be a fiber, then $F$ is quasicompact because $f$ is quasicompact in view of Theorem 38. It follows that $F$ is concentrated. As in the preceding corollary, each point of $F$ is closed. Set $G:=F_{\text {red }}$ and let $x$ be a point of $G$, then $G_{x}$ is a zero-dimensional reduced local ring, whence a field. By Proposition 20, $G$ is an affine scheme. Now $F$ is also an affine scheme by [36, Corollary 8.2]. The conclusion is a consequence of $(1) \Leftrightarrow(2)$.

We recover the result: if $X \rightarrow Y$ is universally closed and injective, then $X \rightarrow Y$ is integral [14-17, Corollaire 18.12.10, page 183], because such a morphism is affine, whence separated.

Consider two morphisms of schemes $f: X \rightarrow Y$, $g: Y \rightarrow S$, such that $g \circ f$ is universally closed and $f$ is surjective. Then $g$ is clearly universally closed. Moreover, if $g$ is separated, it is well known that $f$ is universally closed, whence quasicompact. We deduce from these observations the following result of Raynaud, obtained after a long proof [18, Lemme 3.2].

Proposition 45. Let $f: X \rightarrow Y, g: Y \rightarrow S$ be morphisms of schemes, such that $g \circ f$ is integral, $f$ is surjective, and $g$ is separated. Then $g$ is integral.

Proof. Use Theorem $44(1) \Leftrightarrow(3)$ and the fact that $f$ is quasicompact and then universally subtrusive.

Replace the integral hypothesis on $g \circ f$ with $g \circ f$ is finite in the setting of the above proposition and suppose in addition that $Y$ and $S$ are Noetherian. As the integral and the finite type properties are equivalent to the finite property, we see that $g$ is finite by Theorem 35 .

We end with descent results.

Proposition 46. Let $f: X \rightarrow Y$ be a universally closed surjective morphism of $S$-schemes. If $X / S$ is quasiseparated (separated), then so is $Y / S$.

Proof. The morphism $f \times_{S} f$ is universally closed by [4, I, Proposition 3.8.2], whence quasicompact. From the equation $\left(f \times_{S} f\right) \circ \Delta_{X / S}=\Delta_{Y / S} \circ f$, where the $\Delta_{X / S}$ and $\Delta_{Y / S}$ are the diagonal morphisms of $X / S$ and $Y / S$, we deduce easily that the quasicompactness of $\Delta_{X / S}$ implies the quasicompactness of $\Delta_{Y / S}$ because $f$ is surjective. So the quasiseparated case is proved. The proof of the separated case is similar, since $X / S$ is separated if and only if $\Delta_{X / S}$ is closed.

Note that the quasiseparated case needs only that $f$ be surjective and quasicompact.
Corollary 47. A universally closed morphism $f: X \rightarrow Y$ is concentrated if $Y$ is quasiseparated.

Proof. Use [4, I, Corollaire 6.1.10].

\section{Pure Morphisms}

Hashimoto proved the following result [40, Theorem 1] on pure ring morphisms, that is, universally injective ring morphisms. This result is also a consequence of Theorem 35 .

Proposition 48. Let $S$ be a Noetherian ring, then a pure morphism $A \rightarrow B$ of S-algebras and of finite (presentation) type descends the property $\mathscr{A}$ of S-algebras.

Proof. We note here that $B$ is Noetherian and so is $A$ because $I=I B \cap A$ for each ideal $I$ of $A$. It follows that all the involved algebras are of finite presentation.

We are thus led to consider a class of scheme morphisms introduced by Mesablishvili, that is, a generalization to schemes of the class of pure ring morphisms [41, 42]. We refer the reader to $[41,42]$ for a definition of arbitrary pure morphisms of schemes. We will only consider quasicompact morphisms.

Schematically dominant morphisms of schemes $f: X \rightarrow$ $Y$ are involved. They are defined by $\mathcal{O}_{Y} \rightarrow f_{\star} \mathcal{O}_{X}$ is injective [4, I, Section 5.4] and generalize the injective property of ring morphisms. A Stein morphism $f: X \rightarrow Y$ (such that $\mathcal{O}_{Y} \rightarrow$ $f_{\star} \mathcal{O}_{X}$ is an isomorphism) is schematically dominant.

Let $f: X \rightarrow S$ be a concentrated morphism (quasicompact and quasiseparated). For such a morphism, it is well known that $\mathscr{O}_{S} \rightarrow f_{\star} \mathcal{O}_{X}$ defines a quasicoherent algebra. In view of [4, I, 9.1.21], there is an $S$-morphism of schemes $i_{X}: X \rightarrow X^{o}$, where $X^{o}:=\operatorname{Spec}\left(f_{\star} \mathcal{O}_{X}\right)$ is the relative spectrum of $f$ and $X^{o} \rightarrow S$ is an affine morphism. We can add that $i_{X}$ is Stein, whence schematically dominant.

A schematically dominant morphism is dominant. If $Y$ is reduced, then $f$ is schematically dominant if and only if $f$ is dominant [4, I, Proposition 5.4.3]. The main problem is that the schematically dominant property does not need to be universal. Actually, universally dominant morphisms define pure morphisms, at least for quasicompact morphisms.

Recall that a morphism of schemes is a regular epimorphism if $f$ is a co-equalizer for the projections $p_{1}, p_{2}$ : $X \times_{Y} X \rightarrow X$.

Proposition 49. Let $f: X \rightarrow S$ be a quasicompact morphism of schemes. The following statements are equivalent:

(1) $f: X \rightarrow S$ is pure;

(2) there is an open affine covering $\left(U_{i}\right)_{i \in I}$ of $S$ such that $\mathcal{O}_{S}\left(U_{i}\right) \rightarrow \mathcal{O}_{X}\left(f^{-1}\left(U_{i}\right)\right)$ is a pure ring morphism for each $i$;

(3) $f$ is universally schematically dominant;

(4) $f$ is universally a regular epimorphism.

Proof. We first show that (1) is equivalent to (2). A first step is done by considering [41, Proposition 3.12], which tells us 
that an arbitrary $f$ is pure if and only if there is an affine open cover $\left(U_{i}\right)_{i \in I}$ of $S$ such that $f^{-1}\left(U_{i}\right) \rightarrow U_{i}$ is pure. As $f$ is quasicompact, so are each $f^{-1}\left(U_{i}\right) \rightarrow U_{i}$ and then the result is a consequence of [41, Theorem 5.12 (xi) $\Leftrightarrow$ (xii)]. The rest is [42, Theorem 6.5].

We observe that a composite of quasicompact pure morphisms is pure by Proposition 49(3). If a composite $g \circ$ $f$ of quasicompact morphisms of schemes is pure, so is $g$ [42, Corollary 6.2, Theorem 6.5]. A quasicompact faithfully flat morphism of schemes is pure [41, Remark 3.13]. A quasicompact pure morphism of schemes $f: X \rightarrow S$ is surjective. To see this, use a base change $\kappa(s) \rightarrow S$ for $s \in S$ and Proposition 49(3).

The following result extends to schemes [2, Théorème 37] and add a result to Example 2.

Theorem 50. A quasicompact $f: X \rightarrow Y$ morphism of schemes is universally subtrusive if and only if $X \times_{Y} \operatorname{Spec}(V) \rightarrow \operatorname{Spec}(V)$ is a pure morphism of schemes, for each morphism of schemes $\operatorname{Spec}(V) \rightarrow Y$, where $V$ is a valuation domain. In particular, a quasicompact pure morphism of schemes is universally subtrusive.

Proof. Assume that $f$ is universally subtrusive. By Corollary 8, for each morphism $\operatorname{Spec}(V) \rightarrow Y$, where $V$ is a valuation domain, there is a surjective affine morphism of schemes $g: \operatorname{Spec}\left(V^{\prime}\right) \rightarrow \operatorname{Spec}(V)$ inducing $f$, where $V^{\prime}$ is a valuation domain. We deduce from [2, Proposition $16((1) \Leftrightarrow(3))]$ that $g: \operatorname{Spec}\left(V^{\prime}\right) \rightarrow \operatorname{Spec}(V)$ is pure and so is $X \times_{Y} \operatorname{Spec}(V) \rightarrow \operatorname{Spec}(V)$ that factorizes $g$.

The converse is clear.

We give some examples of descent of the property $\mathscr{A}$, by special pure morphisms that do not involve Noetherian properties

Example 51. (1) We proved that if the $S$-algebra $A$ is a retract of the $S$-algebra $B$ then $A \rightarrow B$ descends $\mathscr{P}$ [43, Lemma 2.3]. Note that in that case $A \rightarrow B$ is a pure ring morphism.

(2) Let $f: A \rightarrow B$ be a pure finite morphism of finite presentation. Then $A \rightarrow B$ descends $\mathscr{A}$. To prove this, first observe that the trace ideal $T_{A}(B)$ of the $A$-module $B$ is $A$ by [44, Lemme 5.5]. Then it is enough to apply [30, Corollary 2].

In particular, if $A$ is a normal ring containing $\mathbb{Q}$, then a finite morphism $A \rightarrow B$ of finite presentation descends $\mathscr{A}$ by [44, Proposition 5.7]. The same result is valid if either $A$ is Prüfer or $A$ is an integrally closed domain $[44,(2)$, (3) page 307].

(3) Let $f: S \rightarrow S^{\prime}$ be a pure ring morphism and $f$ : $A \rightarrow B$ a morphism of $S$-algebras. Then $f$ descends $\mathscr{A},(\mathscr{P})$ if $f \otimes_{S} S^{\prime}$ descends $\mathscr{A},(\mathscr{P})$. The proof is a consequence of [11, Chapitre I, Proposition 5.3] and a remark of [11, page 22].

(4) Let $A \rightarrow B$ be a universally subtrusive ring morphism, where $A$ is a quasi-Prüfer domain; that is, its integral closure $A^{\prime}$ is Prüfer. By using Theorem 50, we get that $A^{\prime} \rightarrow B \otimes_{A} A^{\prime}$ is pure and then a composite $A \rightarrow A^{\prime} \rightarrow$ $B \otimes_{A} A^{\prime}$ of $A \rightarrow B \rightarrow B \otimes_{A} A^{\prime}$, where the first morphism is integral injective and the second is pure. This result is similar to Theorem 4.
We conclude this section by a criterion for a flat morphism of schemes to be schematically dominant, as suggested in [45, Chapter II, Remarques 3.4]. It generalizes the fact that a quasicompact faithfully flat morphism is schematically dominant. We say that a point $x$ of a scheme $X$ is associated with $X$ if the maximal ideal $\mathfrak{m}_{x}$ of $\mathcal{O}_{X, x}$ is the radical of some annihilator $0: a$, where $a \in \mathcal{O}_{X, x}$. This means that $\mathfrak{m}_{x}$ is a weak Bourbaki associated prime ideal of $\mathcal{O}_{X, x}$ (see for instance, [45, Chapter II]), or equivalently, $\mathcal{O}_{X, x}$ is an autoassociated quasi-local ring. We denote by $\operatorname{Ass}(X)$ the set of all associated elements of $X$.

Proposition 52. Let $f: X \rightarrow Y$ be a flat morphism of schemes. Then $f$ is schematically dominant if Ass $(Y) \subseteq f(X)$. The converse holds if in addition $f$ is concentrated.

Proof. Clearly, if $U$ is an open subscheme of $X$, then $\operatorname{Ass}(U)=$ $U \cap \operatorname{Ass}(X)$. As the ring morphism $\mathscr{F}(U) \rightarrow \prod_{x \in U^{F_{x}}}$ is injective for every open subset $U$ of a space $X$ and sheaf $\mathscr{F}$ on $X$, we claim that $\mathcal{O}_{X}(U) \rightarrow \prod_{x \in \operatorname{Ass}(U)} \mathcal{O}_{X, x}$ is injective. Indeed, for an $A$-module $M$ over a ring $A$, we have that $M \rightarrow$ $\prod_{P \in \operatorname{Ass}(M)} M_{P}$ is injective. It follows then that a flat morphism of schemes $f: X \rightarrow Y$ is schematically dominant if $\operatorname{Ass}(Y) \subseteq$ $f(X)$, because $\mathcal{O}_{Y, f(x)} \rightarrow \mathcal{O}_{X, x}$ is faithfully flat.

We prove the converse when $f$ is concentrated. For $y \in$ Ass $(Y)$, consider the flat base change $Z:=\operatorname{Spec}\left(\mathcal{O}_{Y, y}\right) \rightarrow Y$. Then $Z \times_{Y} X \rightarrow Z$ is concentrated, flat, and schematically dense. We thus can assume that $X$ and $Y$ are affine by using Lemma 14, in which case the result follows from [45, Chapter II, Proposition 3.3].

We end by recalling a result of Rydh, which is similar to Proposition 49. Note also that schematically dominant morphisms of $S$-schemes are epimorphisms in the subcategory of separated $S$-schemes, with a converse for concentrated morphisms (see [4, I, 5.4.6]).

Proposition 53 (see [3, Proposition 7.2]). Let $f: X \rightarrow Y$ be a schematically dominant universally submersive morphism of schemes. Then $f$ is an epimorphism in the category of schemes.

It follows that a universally submersive morphism $f$ : $X \rightarrow Y$, with $Y$ reduced is an epimorphism by [4, I, Proposition 5.4.3].

\section{Monomorphisms of Schemes}

The Nagata compactification theorem for schemes says that if $S$ is a concentrated scheme (e.g., any Noetherian scheme) and if $f: X \rightarrow S$ is a separated morphism of finite type of schemes, then $f$ fits into a factorization $f=\bar{f} \circ j$, where $j: X \rightarrow \bar{X}$ is an open immersion and $\bar{f}$ is proper (see [46]). Then $\bar{X}$ is called an $S$-compactification of $X$. Hence, surjective morphisms as above can be factored into an open immersion followed by a universally subtrusive morphism. This justifies that we look at immersions and monomorphisms beyond the fact that they are involved in proofs. 
Recall the following result on monomorphisms, bearing in mind that a morphism of affine schemes is a monomorphism of schemes if and only if its associated ring morphism is an epimorphism of the category of rings. The case of a flat monomorphism is [18, Proposition 1.1].

Lemma 54. Let $f: X \rightarrow Y$ be a morphism of schemes. Then $f$ is a monomorphism of schemes (resp., a flat monomorphism) if and only if $f$ is injective and the ring morphism $\mathcal{O}_{Y, f(x)} \rightarrow$ $\mathcal{O}_{X, x}$ is a ring epimorphism (resp., isomorphism) for each $x \in$ $X$.

Let $f: X \rightarrow Y$ be a morphism of schemes and denote by $\Lambda_{f}$ the class of all pairs of morphisms $u_{1}, u_{2}: Y \rightarrow Z$ equalizing $f$. Then $f$ is called a strict monomorphism if any morphism $g: T \rightarrow X$ such that $\Lambda_{f} \subseteq \Lambda_{g}$ can be uniquely factored $g=f \circ g^{\prime}$. Note that the monomorphism property and the strict monomorphism property are universal.

For instance, let $j: U \rightarrow X$ be an open immersion of schemes $X$, then $j$ is a strict monomorphism; actually $j$ is an equalizer (a kernel) of the double arrow $X \rightarrow X \cup_{U} X$, where $X \cup_{U} X$ is the standard gluing of $X$ and $X$ along $U$ [47, Example 3.1].

Olivier calls immersive a morphism of schemes which is a composite of strict monomorphisms [48]. We have the following results.

Proposition 55 (see [48, Lemme, page 2, Lemme, page 4]). Let $f: X \rightarrow Y$ be a morphism of schemes.

(1) If $f$ is a quasicompact flat monomorphism of schemes, then $f$ is a strict monomorphism, which is quasi-finite and of finite presentation.

(2) If $Y$ is (affine) quasi-local with closed point $y$ such that $f^{-1}(y) \neq \emptyset$, then the following statements are equivalent:

(a) $f$ is immersive;

(b) $f$ is affine and $\mathcal{O}_{Y}(Y) \rightarrow \mathcal{O}_{X}(X)$ is surjective;

(c) $f$ is a strict monomorphism of schemes.

Lemma 56 (see [45, Lemme 1.6]). Let $g: Z \rightarrow Y$ and $f:$ $Y \rightarrow X$ be two morphisms of schemes such that $f$ is separated, $f \circ g$ is a quasicompact flat monomorphism of schemes and $g$ is schematically dominant, then $g$ is a quasicompact flat monomorphism.

Definition 57. Let $\varphi: A \rightarrow B$ be a ring morphism. Then $\varphi$ is called essential if one of the following equivalent conditions is verified.

(1) For each $b \neq 0$ in $B$, there is some $b^{\prime} \in B$ such that $b b^{\prime}=f(a)$ with $a \in A \backslash\{0\}$.

(2) $f^{-1}(J)=0 \Rightarrow J=0$, for each ideal $J$ of $B$.

(3) For each ring morphism $B \rightarrow C$ such that $A \rightarrow C$ is injective, then $B \rightarrow C$ is injective.

An injective integral ring morphism whose target is an integral domain is essential. A flat epimorphism is essential by [45, IV, Proposition 2.1].
Definition 58. Let $f: X \rightarrow Y$ be a morphism of schemes. We say the following.

(1) $f$ is topologically essential if for each nonempty open subset $U$ of $X$, there is an open subset $V$ of $Y$ such that $\emptyset \neq f^{-1}(V) \subseteq U$.

(2) $f$ is a topological immersion if $f$ is injective and if the inverse image topology on $X$ with respect to $f$ coincide with the topology of $X$, or equivalently, $f$ defines a homeomorphism $X \rightarrow f(X)$.

Clearly, a topological immersion is topologically essential. We also note that the notion of topological immersion is dual to the notion of submersive morphism.

Proposition 59. Let $f: X \rightarrow Y$ be a morphism of schemes. The following statements are equivalent.

(1) $f$ is a topological immersion.

(2) $f$ is injective and $f^{-1}(\overline{f(\bar{Z})})=\bar{Z}$ for each subset $Z$ of $X$.

If, in addition, $f$ is quasicompact, then (1) is equivalent to

(3) if $x, x^{\prime} \in X$, then $f(x) \leq f\left(x^{\prime}\right) \Rightarrow x \leq x^{\prime}$.

If $f$ is a topological immersion then $\mathcal{O}_{Y, f(x)} \rightarrow\left(f_{\star} \mathcal{O}_{X}\right)_{f(x)}$ identifies to $\mathcal{O}_{Y, f(x)} \rightarrow \mathcal{O}_{X, x}$ for each $x \in X$.

Proof. It is enough to rework the proof of [7, Proposition 3.20], taking into account that $f(F)$ is proconstructible whenever $F$ is a proconstructible subset of $X$ and $f$ is quasicompact. The end of the proof follows from $[4,0$, 3.4.5].

An immersion of schemes $f: X \rightarrow Y$ is a topological immersion by [4, Section I, 4.2], with $f(X)$ locally closed, open, or closed according to whether $f$ is an immersion, an open immersion, or a closed immersion. A quasicompact flat monomorphism is also a topological immersion by $[3$, Proposition 1.2].

Remark 60. Let $f: \operatorname{Spec}(B) \rightarrow \operatorname{Spec}(A)$ be a morphism of affine schemes, corresponding to a ring morphism $\varphi: A \rightarrow$ $B$.

(1) Then $f$ is topologically essential if and only if for each $b \in B \backslash \operatorname{Nil}(B)$, there is some element $a \in$ $A \backslash \varphi^{-1}(\operatorname{Nil}(B))$ such that $f(a) \in B b$. Assume that $f$ is topologically essential, then so is $f_{\text {red }}$ and we see that $A_{\text {red }} \rightarrow B_{\text {red }}$ is essential. The converse holds if $f$ is dominant.

(2) in [2, Proposition 20, page 545], $\varphi$ is called universally essential if and only if $\varphi$ can be factored $e \circ s$, where $e$ is a flat epimorphism and $s$ is surjective, or also if and only if $A_{P} \rightarrow B_{P}$ is surjective for each $P \in$ $\operatorname{Spec}(A)$, such that $f^{-1}(P) \neq \emptyset$, or also, if and only if $f$ is immersive.

A continuous map of topological spaces $f: X \rightarrow Y$ is called minimal if $f(F) \subset f(X)$ for each closed subset $F \subset X$. This definition is a weakening of injectiveness. 
Proposition 61. Let $f: X \rightarrow Y$ be a dominant morphism of schemes.

(1) If $f$ is topologically essential, then $f$ is a minimal map.

(2) If $f$ is closed and minimal, then $f$ is topologically essential.

Proof. We show (1). Let $F$ be a closed subset of $X$, with $F \neq X$. Then $O:=X \backslash F$ is a nonempty open subset. Since $f$ is topologically essential, let $V$ be an open subset of $Y$ such that $\emptyset \neq f^{-1}(V) \subseteq O$. We get that $V \cap f(F)=\emptyset$ with $V \neq \emptyset$, so that $\overline{f(F)} \subset Y$. This shows that $f$ is minimal. Next we prove (2). Let $O \neq \emptyset$ be an open subset of $X$, then $F:=X \backslash O$ is a closed subset that we can suppose different from $X$. It follows that $f(F)$ is a closed subset of $Y$, different from $Y$. There exists an open subset $V \neq \emptyset$ of $Y$ such that $V \cap f(F)=\emptyset$, so that $f^{-1}(V) \subset O$. As $f$ is surjective, $V=f\left(f^{-1}(V)\right)$ shows that $f^{-1}(V) \neq \emptyset$.

If $f \circ g$ is schematically dominant, then $f$ is clearly schematically dominant. We define a schematically essential morphism of schemes as a morphism of schemes $f: Y \rightarrow S$ such that for each morphism of schemes $g: X \rightarrow Y$ with $f \circ g$ schematically dominant, then $g$ is schematically dominant. Recall that a morphism $f: X \rightarrow Y$ is dominant if maximal points of $Y$ can be lifted up to $X$. The converse holds if $f$ is quasicompact.

Proposition 62. Let $f: Y \rightarrow S$ be a quasicompact morphism of reduced schemes which is a topological immersion, then $f$ is schematically essential.

Proof. We can replace the schematically dominant hypothesis by dominant. Then the result follows from Proposition 59(3). To see this, consider a morphism of schemes $X \rightarrow Y$, such that $f \circ g$ is dominant. Let $y \in Y$ be a maximal point and let $s \in S$ be a maximal point such that $f(y) \leq s$. There is some $x \in X$ such that $f(g(x))=s$, so that $f(y) \leq f(g(x))$. Since $f$ is a topological immersion, we get that $y \leq g(x)$ and finally, $y=g(x)$.

We address the following question. Is the reduced hypothesis necessary in the above proposition? By the way, we have the following result.

Proposition 63. A quasicompact flat monomorphism $f$ : $Y \rightarrow S$ is schematically essential on concentrated flat morphism and on closed immersions.

Proof. First observe that under the hypotheses on $f$, the quasi-local ring $A:=\mathcal{O}_{S, f(y)}$ is auto-associated if $B:=$ $\widehat{O}_{Y, y}$ is auto-associated. Indeed, by Lemma $54, A$ and $B$ are isomorphic. To conclude, use the fact that a flat concentrated morphism $h: Z \rightarrow S$ is schematically dominant if and only if $\operatorname{Ass}(S) \subseteq h(Z)$ by Proposition 52 . The case of closed immersions is [19, Lemme 1.5].

We suspect that the above proposition has a more general version because flat epimorphisms of rings are essential on concentrated morphisms. To see this, suppose that
$X \rightarrow Y \rightarrow S$ is schematically dominant, with $g: X \rightarrow Y$ concentrated and $f: Y \rightarrow S$ a flat monomorphism of affine schemes. We can reduce to the affine case by using a Stein factorization $X \rightarrow X^{\prime} \rightarrow Y$ of $f$, with $X^{\prime} \rightarrow Y$ affine. In that case the result holds [45, IV, Proposition 2.1].

We end by some considerations on quasiaffine schemes. A scheme is called quasiaffine if it is isomorphic to a quasicompact open subscheme of an affine scheme. A morphism $f: X \rightarrow Y$ is called quasiaffine if there exists a covering of $Y$ by affine open subsets $U_{\alpha}$ such that $f^{-1}\left(U_{\alpha}\right)$ is quasiaffine [35, Section 5]. If $X$ is a concentrated scheme with ring of global sections $A$, then $X$ is quasiaffine if and only if $X \rightarrow$ $\operatorname{Spec}(A)$ is an open immersion. In that case, $X \rightarrow \operatorname{Spec}(A)$ is dominant and quasicompact, whence a flat monomorphism of finite presentation.

The following may be useful.

Remark 64. Let $f: X \rightarrow Y$ be a morphism between quasiaffine schemes and set $B:=\mathcal{O}_{X}(X), A:=\mathcal{O}_{Y}(Y)$. Then $f$ is of finite type if $A \rightarrow B$ is a ring morphism of finite type. Indeed, consider the commutative diagram:

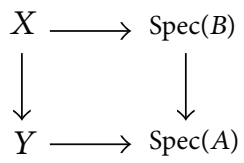

We observe that $X \rightarrow \operatorname{Spec}(B)$ is of finite type, $Y \rightarrow \operatorname{Spec}(A)$ is quasiseparated, and $B \rightarrow A$ is of finite type. Then $X \rightarrow$ $Y$ is of finite type. To see this, consider the composite $X \rightarrow$ $Y \times_{B} A \rightarrow Y$ in the above diagram.

We intend to apply the following result [14-17, Théorème 8.11.2].

Theorem 65. Let $f: X \rightarrow Y$ be a morphism of schemes locally of finite presentation. If $f$ is quasi-finite and separated, then $f$ is quasiaffine. In particular, a quasicompact flat monomorphism is quasiaffine.

Proposition 66. Let $f: X \rightarrow Y$ be a morphism of schemes.

(1) If $f$ is a monomorphism of finite presentation, then $f$ is quasiaffine.

(2) If $f$ is a morphism of S-schemes where $Y$ is locally of finite type over $S$ and quasiseparated, then the graph morphism $\Gamma_{f}: X \rightarrow X \times{ }_{S} Y$ is a quasiaffine monomorphism.

(3) If $f$ is locally of finite type and quasiseparated, then the diagonal morphism $\Delta_{f}: X \rightarrow X \times_{Y} X$ is quasiaffine.

Proof. (1) It is enough to observe that a monomorphism is separated and use the above result.

(2) $\Gamma_{f}$ is a monomorphism since it factors $1_{X}$. Then $p_{X}$ : $X \times_{S} Y \rightarrow X$ is locally of finite type and quasiseparated. In view of [4, Proposition 1.6.3.8(v)], we get that $\Gamma_{f}$ is of finite presentation because $1_{X}=p_{X} \circ \Gamma_{f}$. Hence, $\Gamma_{f}$ is a quasiaffine monomorphism by (1). And (3) follows from (2). 


\section{References}

[1] V. Voevodsky, "Homology of schemes," Selecta Mathematica, vol. 2, no. 1, pp. 111-153, 1996.

[2] G. Picavet, "Submersion et descente," Journal of Algebra, vol. 103, no. 2, pp. 527-591, 1986.

[3] D. Rydh, "Submersions and effective descent of étale morphisms," Bulletin de la Société Mathématique de France, vol. 138, no. 2, pp. 181-230, 2010.

[4] A. Grothendieck, Eléments de géométrie algébrique. I. Le langage des schémas, vol. 166 of Die Grundlehren der Mathematischen Wissenschaften, Springer, 2nd edition, 1971.

[5] J. Kollár, “Quotient spaces modulo algebraic groups," Annals of Mathematics, vol. 145, no. 1, pp. 33-79, 1997.

[6] W. Heinzer and J. Ohm, "Locally Noetherian commutative rings," Transactions of the American Mathematical Society, vol. 158, pp. 273-284, 1971.

[7] D. E. Dobbs, M. Fontana, and G. Picavet, "Generalized goingdown homomorphisms of commutative rings," in Commutative Ring Theory and Applications (Fez, 2001), vol. 231 of Lecture Notes in Pure and Applied Mathematics, pp. 143-163, Dekker, New York, NY, USA, 2003.

[8] M. Nagata, "A theorem on finite generation of a ring," Nagoya Mathematical Journal, vol. 27, pp. 193-205, 1966.

[9] M. Nagata and K. Otsuka, "Some remarks on the 14th problem of Hilbert," Journal of Mathematics of Kyoto University, vol. 5, no. 1, pp. 61-66, 1965.

[10] A. Constantinescu, "Schemes dominated by algebraic varieties and some classes of scheme morphisms. I," Acta Universitatis Apulensis, no. 16, pp. 37-51, 2008.

[11] J. P. Olivier, "Descente de quelques propriétés élémentaires par morphismes purs," Anais da Academia Brasileira de Ciências, vol. 45, pp. 17-33, 1973.

[12] http://mathoverflow.net/questions/23337/is-a-universallyclosed-morphism-of-schemes-quasi-compact.

[13] Chapter: Morphisms of schemes, http://stacks.math.columbia .edu .

[14] A. Grothendieck, "Éléments de géométrie algébrique. IV. Étude locale des schémas et des morphismes de schémas. I," Publications Mathématiques de l'Institut des Hautes Études Scientifiques, no. 20, 1964.

[15] A. Grothendieck, "Éléments de géométrie algébrique. IV. Étude locale des schémas et des morphismes de schémas. II," Publications Mathématiques de l'Institut des Hautes Études Scientifiques, no. $24,1965$.

[16] A. Grothendieck, "Éléments de géométrie algébrique. IV. Étude locale des schémas et des morphismes de schémas. III," Publications Mathématiques de l'Institut des Hautes Études Scientifiques, no. $28,1966$.

[17] A. Grothendieck, "Éléments de géométrie algébrique. IV. Étude locale des schémas et des morphismes de schémas IV," Publications Mathématiques de l'Institut des Hautes Études Scientifiques, no. 32, 1967.

[18] M. Raynaud, "Un critère d'effectivité de descente," in Séminaire d'Algèbre Commutative 1967-1968, Secrétariat Mathématique, exposé no 5, 1968.

[19] D. Ferrand, "Monomorphismes et morphismes absolument plats," Bulletin de la Société Mathématique de France, vol. 100, pp. 97-128, 1972.

[20] M. Raynaud and L. Gruson, "Critères de platitude et de projectivité," Inventiones Mathematicae, vol. 13, pp. 1-89, 1971.
[21] J. P. Olivier, "Anneaux absolument plats universels et épimorphismes à buts réduits," in Séminaire d'Algèbre commutative 1967-1968, Secrétariat Mathématique, exposé no 6, 1968.

[22] M. Hochster, "Prime ideal structure in commutative rings," Transactions of the American Mathematical Society, vol. 142, pp. 43-60, 1969.

[23] D. Ferrand, "Conducteur, descente et pincement," Bulletin de la Société Mathématique de France, vol. 131, no. 4, pp. 553-585, 2003.

[24] G. Picavet, "Seminormal or $t$-closed schemes and Rees rings," Algebras and Representation Theory, vol. 1, no. 3, pp. 255-309, 1998.

[25] N. Onoda, "Subrings of finitely generated rings over a pseudogeometric ring," Japanese Journal of Mathematics, vol. 10, no. 1, pp. 29-53, 1984.

[26] J. M. Giral, "Krull dimension, transcendence degree and subalgebras of finitely generated algebras," Archiv der Mathematik, vol. 36, no. 4, pp. 305-312, 1981.

[27] J. Fogarty, "Geometric quotients are algebraic schemes," Advances in Mathematics, vol. 48, no. 2, pp. 166-171, 1983.

[28] J. Alper, "Fogarthy's proof of the finite generation of certain subrings," Expository paper, http://maths-people.anu.edu.au/ alperj/.

[29] M. Hashimoto, “Geometric quotients are algebraic schemes' based on Fogarty's idea," Journal of Mathematics of Kyoto University, vol. 43, no. 4, pp. 807-814, 2003.

[30] M. Lorenz, "On affine algebras," in Ring Theory, vol. 1197 of Lecture Notes in Mathematics, pp. 121-126, Springer, Berlin, Germany, 1986.

[31] D. Gale, "Subalgebras of an algebra with a single generator are finitely generated," Proceedings of the American Mathematical Society, vol. 8, pp. 929-930, 1957.

[32] I. J. Papick, "Affine pairs," in Rings, Modules, Algebras, and Abelian Groups, vol. 236 of Lecture Notes in Pure and Applied Mathematics, pp. 437-448, Dekker, New York, NY, USA, 2004.

[33] Chapter: More on morphisms, http://stacks.math.columbia.edu

[34] Q. Liu, Algebraic Geometry and Arithmetic Curves, vol. 6 of Oxford Graduate Texts in Mathematics, Oxford Science Publications, Oxford University Press, Oxford, UK, 2002.

[35] A. Grothendieck, "Éléments de géométrie algébrique. II. Étude globale élémentaire de quelques classes de morphismes," Publications Mathématiques de l'Institut des Hautes Études Scientifiques, no. 8, 1961.

[36] D. Rydh, "Noetherian approximation of algebraic spaces and stacks," http://arxiv.org/abs/0904.0227v2.

[37] Chapter: Properties of schemes, http://stacks.math.columbia .edu.

[38] J. P. Olivier, "Montée des propriétés par morphismes absolument plats," in Comptes-Rendus des Journées d'Algèbre Pure et Appliquée. Montpellier, pp. 86-109, Université des Sciences et Techniques du Languedoc, Montpellier, France, 1971.

[39] U. Görtz and T. Wedhorn, Algebraic Geometry I: Schemes with Examples and Exercises, Advanced Lectures in Mathematics, Vieweg and Teubner, Wiesbaden, Germany, 2010.

[40] M. Hashimoto, "A pure subalgebra of a finitely generated algebra is finitely generated," Proceedings of the American Mathematical Society, vol. 133, no. 8, pp. 2233-2235, 2005.

[41] B. Mesablishvili, "Descent theory for schemes," Applied Categorical Structures, vol. 12, no. 5-6, pp. 485-512, 2004. 
[42] B. Mesablishvili, "More on descent theory for schemes," Georgian Mathematical Journal, vol. 11, no. 4, pp. 783-800, 2004.

[43] G. Picavet, "Algebraically flat or projective algebras," Journal of Pure and Applied Algebra, vol. 174, no. 2, pp. 163-185, 2002.

[44] G. Picavet, "Pureté, rigidité et morphismes entiers," Transactions of the American Mathematical Society, vol. 323, no. 1, pp. 283-313, 1991.

[45] D. Lazard, "Autour de la platitude," Bulletin de la Société Mathématique de France, vol. 97, pp. 81-128, 1969.

[46] B. Conrad, M. Lieblich, and M. Olson, "Nagata compactification for algebraic spaces," Journal de l'Institut de Mathématiques de Jussieu, vol. 11, pp. 747-814, 2012.

[47] K. Schwede, "Gluing schemes and a scheme without closed points," in Recent Progress in Arithmetic and Algebraic Geometry, vol. 386 of Contemporary Mathematics, pp. 157-172, American Mathematical Society, Providence, RI, USA, 2005.

[48] J. P. Olivier, Morphismes Immergeants de Ann. U.E.R. de Mathématiques, 1970-1971, Secrétariat des Mathématiques, Publication No 106, Université des Sciences et Techniques du Languedoc, Montpellier, France, 1971. 


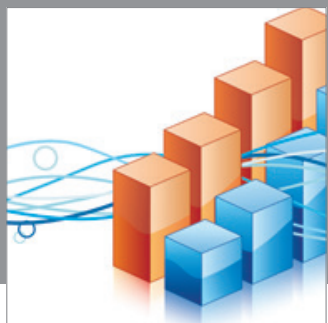

Advances in

Operations Research

mansans

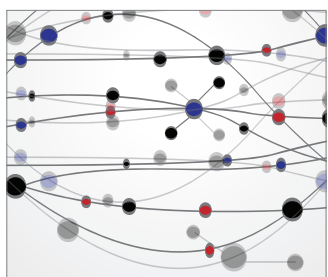

The Scientific World Journal
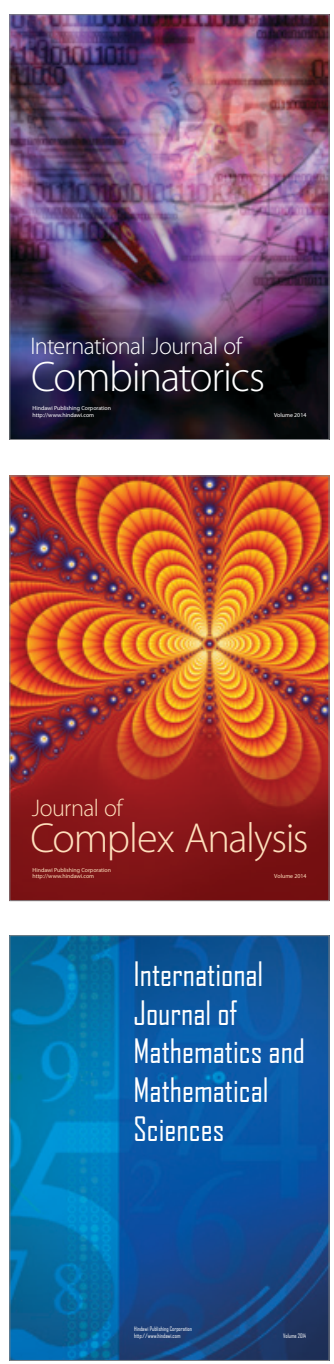
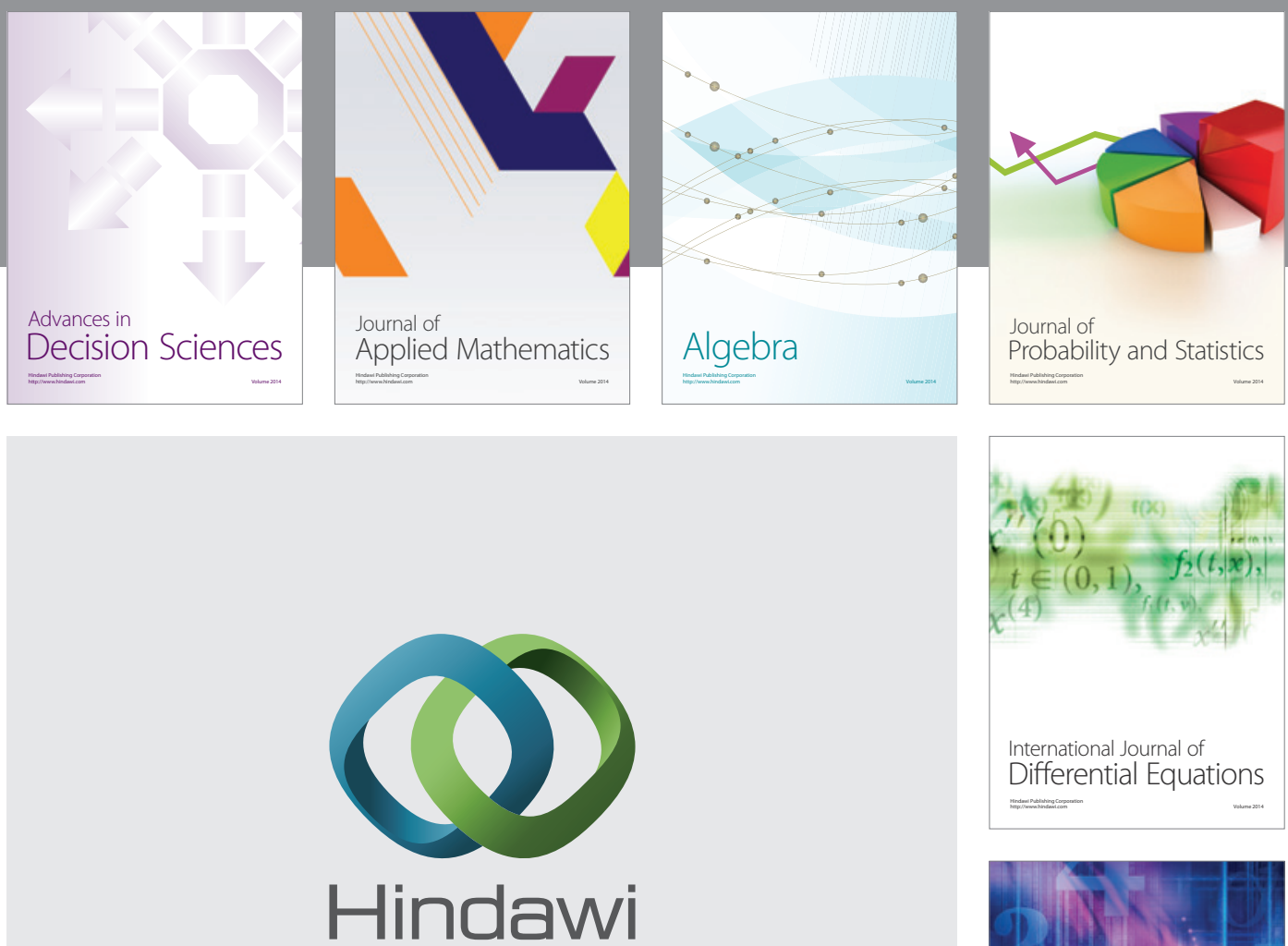

Submit your manuscripts at http://www.hindawi.com
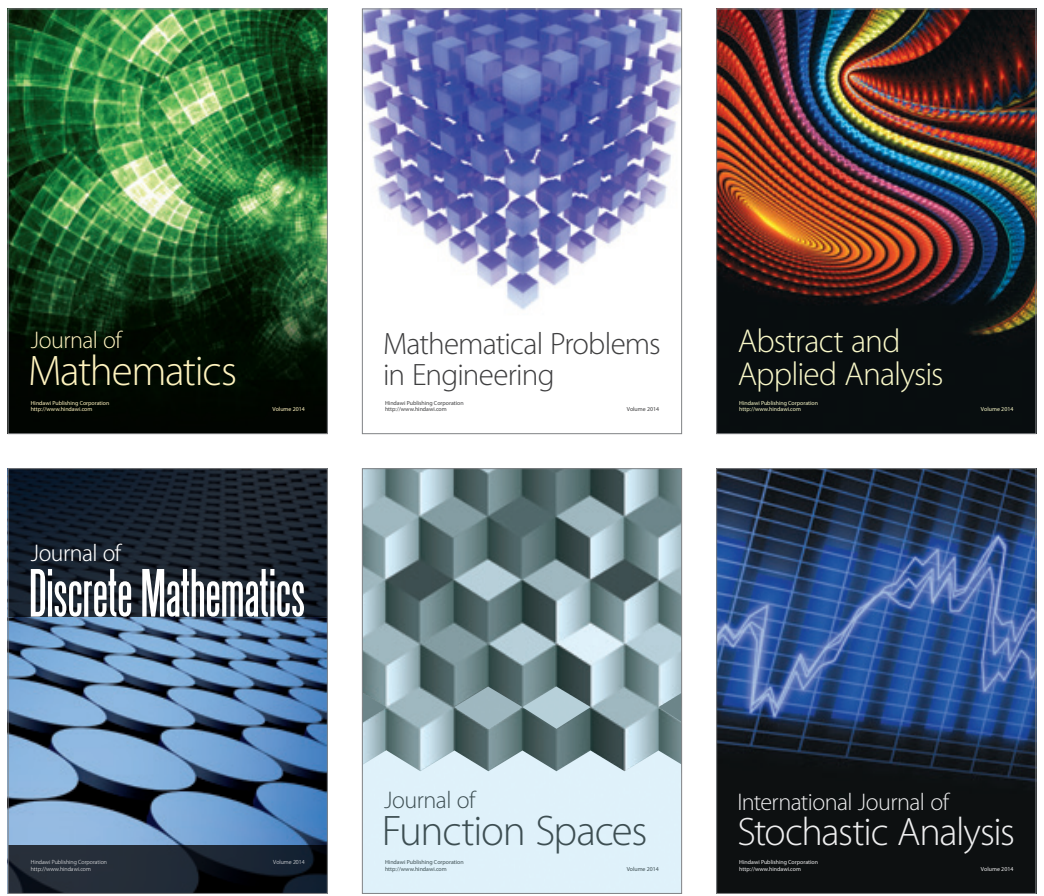

Journal of

Function Spaces

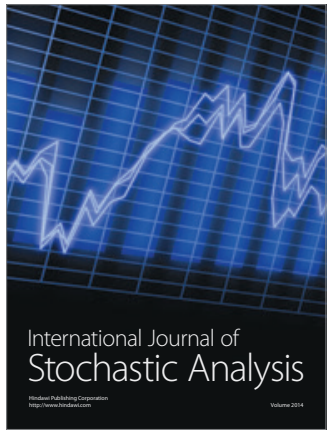

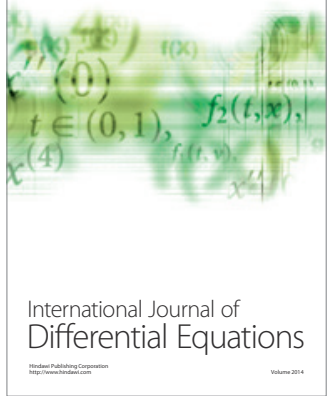
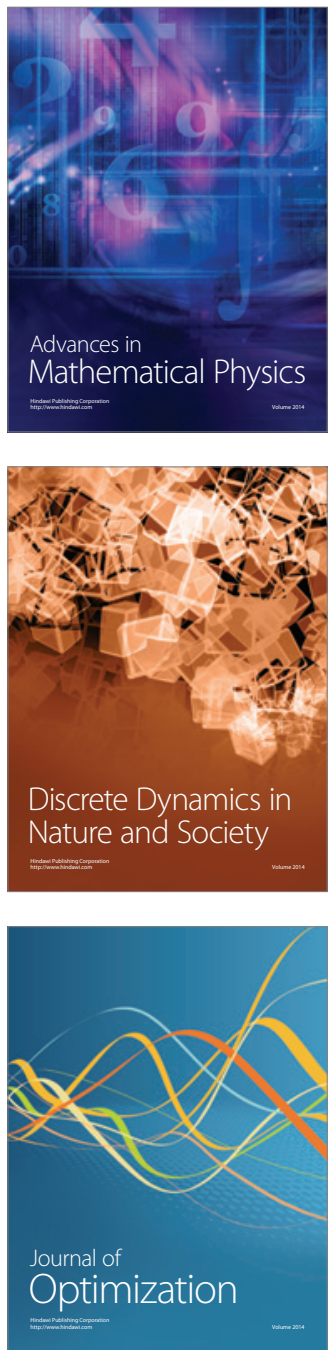